\title{
Validity of HAT Score for Predicting Symptomatic Intracranial Hemorrhage in Acute Stroke Patients with Proximal Occlusions: Data from Randomized Trials of Sonothrombolysis
}

\author{
Georgios Tsivgoulis $^{a}$ e Maher Saqqur $^{c}$ Andrew Barreto $^{b}$ Andrew M. Demchuk ${ }^{d}$ \\ Marc Ribo $^{g}$ Marta Rubierag Vijay K. Sharma ${ }^{\text {h }}$ Elefterios Stamboulis $^{f}$ \\ Peter D. Schellinger ${ }^{i}$ Carlos A. Molinag Andrei V. Alexandrov ${ }^{a}$ \\ ${ }^{a}$ Comprehensive Stroke Center, University of Alabama at Birmingham Hospital, Birmingham, Ala., and \\ ${ }^{\mathrm{b}}$ Stroke Program, The University of Texas-Houston Medical School, Houston, Tex., USA; ' $D i v i s i o n$ of Neurology, \\ University of Alberta Medical School, Edmonton, Alta., and d Calgary Stroke Program, Department of Clinical

 \\ Thrace, Alexandroupolis, and fSecond Department of Neurology, University of Athens, School of Medicine, \\ Athens, Greece; ${ }^{9}$ Department of Neurology, Hospital Universitari Vall d'Hebron, Universitat Autonoma de Barcelona, \\ Barcelona, Spain; h Division of Neurology, Department of Medicine, National University Hospital, Singapore; \\ 'Department of Neurology, University Hospital of Erlangen, Erlangen, Germany
}

\section{Key Words}

Thrombolysis • Symptomatic intracranial hemorrhage • Stroke $\cdot$ Occlusion

\begin{abstract}
Background: The Hemorrhage after Thrombolysis (HAT) score has recently been introduced as a practical scale for risk stratification of intracranial hemorrhage $(\mathrm{ICH})$ in patients receiving intravenous tissue plasminogen activator (tPA). We aimed to externally validate and evaluate the predictive ability of the HAT score in patients with proximal arterial occlusions (PAO) enrolled into randomized clinical trials of sonothrombolysis. Methods: The HAT score (range 0, minimum risk, to 5, maximum risk) was retrospectively calculated for each patient using clinical trial data (baseline NIHSS, extent of hypodensity on $\mathrm{CT}$, history of diabetes mellitus and serum glucose). Symptomatic ICH ( $\mathrm{IICH}$ ) was defined as imaging evidence of $\mathrm{ICH}$ with clinical worsening (NIHSS $\geq 4$ )
\end{abstract}

within $72 \mathrm{~h}$ from stroke onset. The predictive ability of the HAT score for sICH and any ICH (both asymptomatic and symptomatic) was calculated using c statistics. Results: A total of 161 tPA-treated patients (mean age $68 \pm 13$ years, 58\% men, median NIHSS 16, interquartile range 9) with PAO were randomized in TUCSON $(n=35)$ and CLOTBUST $(n=126)$. sICH occurred in 9 (5.6\%) cases, and 6 had asymptomatic ICH. The rates of sICH for the corresponding HAT scores were: HAT 0-1: 3\%; 2: 9\%; 3: 14\%; 4-5: 14\%. The risk of sICH (c statistic $0.72,95 \% \mathrm{Cl}: 0.58-0.86 ; \mathrm{p}=0.027$ ) and any ICH (c statistic 0.70, 95\% Cl: 0.58-0.82; $p=0.011$ ) increased with higher HAT scores. Higher HAT scores were also associated with higher likelihood of persisting occlusion (c statistic $0.63,95 \% \mathrm{Cl}$ : 0.54-0.72; $p=0.004$ ). Conclusions: The HAT score has reasonable external validity for predicting the risk of $\mathrm{sICH}$ following intravenous thrombolysis in patients with PAO. Moreover, higher HAT scores appear to be associated with higher likelihood of persisting occlusion in tPA-treated patients.

Copyright $\odot 2011$ S. Karger AG, Basel

\section{KARGER}

Fax +41613061234 E-Mail karger@karger.ch www.karger.com

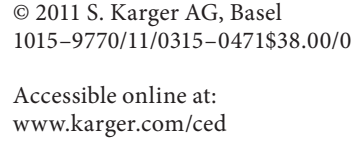

Dr. Georgios Tsivgoulis

Department of Neurology, Democritus University of Thrace

Kapodistriou 3, Nea Xili

GR-68100 Alexandroupolis (Greece)

Tel. +30 693717 8635, E-Mail tsivgoulisgiorg@yahoo.gr 


\section{Introduction}

Symptomatic intracranial hemorrhage (sICH) is the most unfavorable and feared complication of intravenous thrombolysis and the most important factor deterring the use of intravenous tissue plasminogen activator (ivtPA) in patients with acute ischemic stroke [1]. The most consistently identified predictors of sICH in acute revascularization trials using thrombolytic therapies have been the dose of lytic agents, edema or mass effect on head CT and stroke severity [2]. Other risk factors that may be associated with sICH include older age, hyperglycemia, concurrent heparin use, timing of therapy, and timing of recanalization $[1,2]$.

The Hemorrhage after Thrombolysis (HAT) score has recently been introduced as a practical scale for risk stratification of intracranial hemorrhage (ICH) in patients receiving iv-tPA [3]. The main advantages of this practical scale are related to its wide applicability and the easiness to perform since the computation of the score is based on baseline stroke severity, extent of hypodensity on admission CT scan and baseline serum glucose or history of diabetes mellitus. However, its predictive ability has not been externally validated or tested in patients with acute proximal arterial occlusions. We aimed to externally validate HAT scores in patients with acute proximal intracranial arterial occlusions enrolled in randomized clinical trials of sonothrombolysis $[4,5]$.

\section{Methods}

We retrospectively analyzed data collected from patients who were enrolled in CLOTBUST (Combined Lysis of Thrombus in Brain ischemia using transcranial Ultrasound and Systemic TPA) [4] and TUCSON (Transcranial Ultrasound in Clinical SONothrombolysis) [5], two phase II trials of ultrasound-enhanced thrombolysis. Patients were considered eligible for CLOTBUST and TUCSON only when they had transcranial Doppler (TCD) evidence of an acute proximal intracranial arterial occlusion. In both trials, arterial occlusions were diagnosed using Thrombolysis in Brain Ischemia (TIBI) flow grades [6]. TIBI flow grades were previously validated against invasive angiography with an excellent $(90-100 \%)$ agreement, particularly for the proximal middle cerebral artery (MCA) occlusion location as well as determining persisting occlusion, partial or complete recanalization [6-12]. Pretreatment stroke severity was evaluated in both trials using the NIH Stroke Scale Score (NIHSS score), while blood glucose values were measured in all patients at hospital admission. All patients received standard iv-tPA therapy $(0.9 \mathrm{mg} / \mathrm{kg}$ dose, maximum 90 $\mathrm{mg}, 10 \%$ bolus, $90 \%$ continuous infusion for $1 \mathrm{~h}$ following bolus injection) that was initiated within the first $3 \mathrm{~h}$ after symptom onset according to published criteria [13].
The HAT score (range 0, minimum risk, to 5, maximum risk) was retrospectively calculated for each patient using prospectively collected data for the following clinical and neuroimaging variables: pretreatment NIHSS (<15: 0 point; $15-20$ : 1 point; $>20: 2$ points), history of diabetes mellitus or baseline serum glucose $>200 \mathrm{mg} / \mathrm{dl}$ upon admission (1 point) and presence of early visible hypodensity on initial brain CT scan (absence of hypodensity: 0 point, $<1 / 3$ of MCA territory: 1 point; $\geq 1 / 3$ MCA territory: 2 points) [3].

sICH was diagnosed using the definition that was adopted by investigators prior to clinical trial initiation: imaging evidence of ICH with clinical worsening (NIHSS increase of 4 or more points from baseline assessment) within $72 \mathrm{~h}$ from stroke onset [4]. In case of doubt with regard to whether edema or hemorrhage was the leading pathology causing the clinical deterioration (increase of $\geq 4$ points in NIHSS score), an association of the hemorrhage with deterioration was invariably assumed in order to avoid missing any sICH cases, since CLOTBUST and TUCSON were phase II safety and feasibility trials. Consequently, sICH was diagnosed in all patients with a clinical deterioration of 4 or more points in the NIHSS score and brain imaging evidence of $\mathrm{ICH}$, independent of the presence of causality between clinical deterioration and $\mathrm{ICH}$ in each case. There was no central adjudication for $\mathrm{sICH}$ in CLOTBUST [4]. The treating physicians at the four centers, who were experienced stroke neurologists, made this diagnosis. In TUCSON a site investigator blinded to the treatment allocation made the diagnosis of sICH. A central reader who was also blinded to the treatment group confirmed all cases. In addition, the central reader reviewed the baseline and repeat CT scans of all patients enrolled in the TUCSON trial in order to detect the presence of any intracranial bleed that may have been missed by the site investigator [5].

Asymptomatic ICH (aICH) was defined as imaging evidence of ICH (hemorrhagic transformation or parenchymal hematoma) without clinical worsening (NIHSS increase of 3 or less points from baseline assessment) [5]. Persisting occlusion, partial and complete recanalization were diagnosed on the basis of our validated TIBI flow grading system at the end of TCD monitoring as previously described [6-12]. Patients with reocclusion during TCD monitoring were diagnosed as having persisting occlusion at the end of TCD monitoring [4, 5, 14]. Favorable functional outcome was defined as a modified Rankin Scale score of $0-2$ at 3 months after treatment [3-5]. Further details regarding the protocols of both trials have previously been published $[15,16]$. In order to be able to make direct comparisons with previous studies we also used the definition of the Safe Implementation of Thrombolysis in Stroke Monitoring Study (SITS-MOST) for sICH and repeated all analyses [17].

\section{Statistical Analyses}

Statistical comparisons were performed between patient groups stratified by the HAT score using the $\chi^{2}$ test, Fisher's exact test, unpaired t test, and Mann-Whitney $U$ test as indicated for dichotomous or continuous variables. The adjusted Wald method, which provides the best coverage for binomial confidence intervals (CI) when samples are less than 150 [18], was used for computation of $95 \% \mathrm{CI}$ for the sICH rates across the different HAT-score subgroups. We evaluated the predictive ability of the score for detection of sICH, any ICH (combination of aICH and sICH), persisting occlusion and favorable functional outcome at 
Table 1. Baseline characteristics, ultrasonographic and neuroimaging findings of the study population $(n=161)$

\begin{tabular}{lc}
\hline Baseline characteristics & \\
\hline Mean age (SD), years & $68(13)$ \\
TUCSON, $\mathrm{n}$ & $35(22 \%)$ \\
CLOTBUST, $\mathrm{n}$ & $126(78 \%)$ \\
Male gender, $\mathrm{n}$ & $93(58 \%)$ \\
Diabetes mellitus, $\mathrm{n}$ & $39(24 \%)$ \\
Median baseline NIHSS (IQR), points & $16(9)$ \\
Median baseline serum glucose (IQR), mg/dl & $121(55)$ \\
Median HAT (IQR) & $1(2)$ \\
sICH, n & $9(5.6 \%)$ \\
aICH, $\mathrm{n}$ & $6(3.7 \%)$ \\
Sustained complete recanalization ${ }^{1}, \mathrm{n}$ & $53(33 \%)$ \\
Persisting occlusion, $\mathrm{n}$ & $64(40 \%)$ \\
\hline
\end{tabular}

$\mathrm{IQR}=$ Interquartile range. ${ }^{1}$ At the end of TCD monitoring.

3 months using receiver operating characteristic curve models. In addition, accuracy parameters (sensitivity, specificity, positive predictive value (PPV), negative predictive value (NPV) and overall accuracy) were determined at each cutoff of the score for the different outcome variables. Areas under receiver-operator curves (c statistic) and corresponding 95\% CIs were calculated as a measure of predictive ability. The c statistic integrates sensitivity and specificity of the range of a variable, and estimates how well a prediction rule can correctly rank-order patients by risk. Ideal prediction produces a c statistic of 1.00; prediction no better than chance is associated with a c statistic of $\leq 0.50$. The Statistical Package for Social Science (SPSS Inc., version 13.0 for Windows) was used for statistical analyses.

\section{Results}

A total of 161 iv-tPA-treated patients (mean age $68 \pm$ 13 years, 58\% men, median NIHSS 16, interquartile range 9) with proximal arterial occlusions were enrolled in TUCSON $(n=35)$ and CLOTBUST $(n=126)$. The baseline characteristics, the ultrasonographic and neuroimaging findings of the study population are summarized in table 1 . Sustained complete recanalization was present in 53 patients at the end of TCD monitoring (33\%), while persisting occlusion was identified in 64 cases (40\%). sICH occurred in 9 (5.6\%) cases (6 in CLOTBUST and 3 in TUCSON), and 6 patients (3.7\%) had aICH (3 in CLOTBUST and 3 in TUCSON). When the SITS-MOST definition was used for the documentation of sICH, the same 9 cases also met SITS-MOST criteria for symptomatic intracranial bleeding. The rate of sICH was similar in the
Table 2. Risk of sICH stratified according to HAT scores in the study population $(\mathrm{n}=161)$

\begin{tabular}{lll}
\hline HAT score $^{1}$ & sICH, $\mathrm{n}$ & sICH risk, \% $\left(95 \% \mathrm{CI}^{2}\right)$ \\
\hline $0-1(\mathrm{n}=102)$ & 3 & $3(0-9)$ \\
$2(\mathrm{n}=45)$ & 4 & $9(0-21)$ \\
$3(\mathrm{n}=7)$ & 1 & $14(0-53)$ \\
$4-5(\mathrm{n}=7)$ & 1 & $14(0-53)$ \\
\hline
\end{tabular}

${ }^{1} \mathrm{p}$ for linear trend $=0.024$.

${ }^{2}$ Computed by the adjusted Wald method [14].

Table 3. Predictive ability of HAT scores for detecting sICH, any $\mathrm{ICH}$, persisting occlusion and favorable functional outcome at 3 months

\begin{tabular}{llc}
\hline Variable & c statistic $(95 \% \mathrm{CI})$ & $\mathrm{p}$ value \\
\hline sICH & $0.72(0.58-0.86)$ & 0.027 \\
Any ICH & $0.70(0.58-0.82)$ & 0.011 \\
Persisting occlusion & $0.63(0.55-0.72)$ & 0.004 \\
Favorable functional & & \\
$\quad$ outcome at 3 months & $0.76(0.68-0.84)$ & $<0.001$ \\
Death at 3 months & $0.69(0.59-0.80)$ & 0.005 \\
\hline
\end{tabular}

group receiving sonothrombolysis $(7 \%, 6 / 86)$ and iv-tPA alone $(4 \%, 3 / 75 ; \mathrm{p}=0.505$ by Fisher's exact test). The median HAT score was 1 (interquartile range 2 ). The median HAT score did not differ between the ultrasound-enhanced thrombolysis (1, interquartile range 2$)$ and intravenous thrombolysis groups ( 1 , interquartile range $1 ; \mathrm{p}=$ 0.779 by Mann-Whitney U test).

The rates of sICH for the corresponding HAT scores are shown in table 2: HAT 0-1:3\%; 2: 9\%; 3: 14\%; 4-5: 14\% ( $\mathrm{p}$ for linear trend $=0.024$ ). The actual numbers of $\mathrm{sICH}$ in the respective HAT-score categories were as follows: 0 points (0/44), 1 point (3/58), 2 points (4/45), 3 points $(1 / 7)$, 4 points (1/6), 5 points (0/1). The risk of sICH increased with higher HAT scores (c statistic 0.72, 95\% CI: 0.58$0.86 ; \mathrm{p}=0.027$; table 3 ). Similarly, the risk of any $\mathrm{ICH}$ was associated with higher HAT scores (c statistic 0.70, 95\% CI: $0.58-0.82 ; \mathrm{p}=0.011$; table 3 ). A HAT score of $>1$ for predicting sICH had a sensitivity of $66 \%$, a specificity of $65 \%$, a PPV of $10 \%$, a NPV of $97 \%$ and an overall accuracy of $65 \%$ (6 true positive, 3 false negative, 53 false positive and 99 true negative cases). A higher threshold (>2) had optimal accuracy parameters with higher overall ac- 
curacy (88\%), specificity (92\%) and PPV (14\%), similar NPV (95\%) and lower sensitivity (22\%).

There were 6 cases with hypodensity $\geq 1 / 3$ MCA territory on their baseline CT scan, and 1 sICH (17\%) occurred in this subgroup. Since visible hypodensity exceeding $1 / 3$ of the MCA territory is considered a contraindication to thrombolysis in many centers, we repeated our analyses after excluding these 6 patients. The predictive ability of the HAT score for detecting sICH remained practically unchanged (c statistic $0.70,95 \%$ CI: $0.56-0.82$; $\mathrm{p}=0.046)$.

Higher HAT scores were also found to be predictive of persisting occlusion (c statistic 0.63 , 95\% CI: 0.54-0.72; $\mathrm{p}=0.004$; table 3 ). Finally, the HAT score reasonably predicted favorable functional outcome at 3 months (c statistic 0.76, 95\% CI: 0.68-0.84; $\mathrm{p}<0.001$; table 3). Higher HAT scores were also predictive of death at 3 months (c statistic $0.69,95 \%$ CI: 0.59-0.80; $\mathrm{p}=0.005$; table 3 ).

\section{Discussion}

We attempted to evaluate the external validity of the HAT score using prospectively and independently collected sICH data from two phase II randomized trials of sonothrombolysis. Interestingly, we documented similar c statistics to those reported in the study [3] introducing the HAT score for all outcome variables including sICH (0.72 vs. 0.74$)$, any ICH ( 0.70 vs. 0.72$)$ and 3 -month favorable functional outcome ( 0.76 vs. 0.75 ). The present study findings are also in line with the results of a Spanish study that has recently been presented at the International Stroke Conference 2010 [19]. The investigators retrospectively analyzed data from a prospective registry of patients treated with TPA in two centers. They documented that higher HAT scores were independently associated with higher likelihood of sICH (OR: 1.5, 95\% CI: 1.1-2.0; $\mathrm{p}=0.005)$, hemorrhagic transformation (OR: $1.8,95 \% \mathrm{CI}$ : $1.5-2.1 ; \mathrm{p}<0.001)$ and poor functional outcome (OR: 3.0, 95\% CI: 2.4-3.7; $\mathrm{p}<0.001)$ defined as a modified Rankin Scale score of $>2$. Unfortunately, the predictive ability of the HAT score was not evaluated using $c$ statistics and therefore their data cannot be directly compared to our dataset.

Given the fact that all elements comprising the HAT score have previously been associated with a higher likelihood of persisting occlusion [20-25], we also investigated the potential relationship of this risk stratification scale with the odds of persisting occlusion on TCD monitoring during the first $2 \mathrm{~h}$ following a tPA bolus. The de- tected association of higher HAT scores with an increased risk of persisting occlusion is in full agreement with previous observations from our collaborative group. More specifically, higher NIHSS scores represent a higher clot burden and have been repeatedly documented to substantially decrease the likelihood of recanalization in patients treated with intravenous thrombolysis [4]. In addition, hyperglycemia is a well-known inhibitor of fibrinolysis [26] and it has been constantly shown to hamper recanalization, thus increasing the likelihood of persisting occlusion in different iv-tPA registries monitoring recanalization in real time with TCD [21, 22]. Finally, early ischemic changes on brain CT scans quantified by the Alberta Stroke Program Early CT Score have previously been associated with lower likelihood of complete recanalization and functional independence following intravenous thrombolysis $[23,25]$.

Our findings indicate that the potential clinical applicability of the HAT score may be related to its high NPV (95\%) and specificity (92\%) for ruling out sICH in patients with HAT scores less than 3. Consequently, it may serve as a complementary tool to clinical bedside evaluation for the selection of acute ischemic stroke patients with minimal risk of symptomatic intracranial bleeding following intravenous thrombolysis, especially in stroke centers with limited experience with tPA treatment. In contrast, the poor sensitivity and PPV of the HAT score suggest that potential candidates for iv-tPA should not be excluded from this treatment solely on the basis of high HAT scores. More specifically, in our series the rate of sICH was $14 \%$ in patients with HAT score $>3$, while in the study introducing the HAT score the risk was 3 times higher (42\%), indicating that patients in this specific range of the HAT score (4-5) carry an unacceptable risk of symptomatic intracranial bleeding following intravenous thrombolysis [3]. The discrepant findings between the two studies in this specific subgroup of patients (HAT score: 4-5) may be related to the limited sample size both in our $(\mathrm{n}=$ $7)$ and the combined NINDS/Boston $(n=9)$ cohort and call for independent validation in larger datasets. Nevertheless, the high specificity and NPV documented in both studies highlight that calculation of the HAT score in everyday clinical practice may offer reassurance to reluctant and relatively inexperienced physicians that patients with HAT scores $\leq 2$ carry low risk for sICH and this may facilitate the wider implementation of intravenous thrombolysis, especially in community centers.

Certain limitations of the present report need to be acknowledged. For one, this is a retrospective analysis of prospectively collected data. In addition, our sample size 
is moderate and further independent validation of this score in larger cohorts of patients is required before considering its potential application in clinical practice. More specifically, some of the HAT scores, especially $\geq 3$ points, had very few patients in each category, suggesting that a great deal of imprecision may exist at these levels, with potential variance due to case misclassification. In addition and perhaps related to the previous point about low numbers of observations, the rate of sICH (14\%) was the same in the HAT group with 3 points and with 4-5 points, suggesting that some of the parameters included in the score may not add substantially to its predictive value or that a ceiling effect may exist with higher HAT scores.

Also, our study population encompasses patients with proximal arterial occlusions; therefore our results cannot be generalized to patients with distal arterial occlusions or lacunar strokes. It should also be noted that the accuracy parameters of TCD are less reliable than the gold standard (digital subtraction angiography) and are largely dependent on the experience and training of the sonographers [27]. Besides, the reported rate of aICH (3.7\%) is low compared to other studies and this may be related to the fact that $\mathrm{T}_{2}$-weighted MRI was not used for ICH detection and therefore some subtle aICHs may have been missed on conventional CT scans. Another potential explanation may be related to the lack of central adjudication of brain CT scan interpretation in CLOTBUST where only 3 aICHs were detected out of a sample of 126 subjects.

In conclusion, our study externally validates the predictive ability of the HAT score for detecting sICH (inde- pendent of the definition used) in a subgroup of patients with acute proximal intracranial occlusions treated with iv-tPA. Additionally, higher HAT scores were also predictive of persisting occlusion at the end of TCD monitoring and poor functional outcome at 3 months following onset. Although the former association is intriguing, it may serve only for hypothesis generation and warrants further independent validation in prospective iv-tPA registries.

\section{Disclosure Statement}

Dr. Tsivgoulis received a fellowship grant from the Neurology Department, Eginition Hospital, University of Athens School of Medicine, Athens, Greece. Dr. Tsivgoulis and Dr. Alexandrov had full access to all data in the study and take responsibility for the integrity of the data and the accuracy of data analysis. Dr. Saqqur was the Principal Investigator at Edmonton site for the CLOTBUST trial. Dr. Barreto was the Principal Investigator at Houston site for the TUCSON trial. Dr. Demchuk was the Principal Investigator at Calgary site for the CLOTBUST trial. Dr. Ribo none. Dr. Rubiera received a fellowship grant from the Instituto de Salud Carlos III and Institut de Recerca Hospital Vall d'Hebron, Barcelona, Spain. Dr. Sharma none. Dr. Stamboulis none. Dr. Schellinger received speaker honoraria from Boehringer Ingelheim, the manufacturer of rt-PA and served as a consultant for ImaRx Therapeutics, Inc. Dr. Molina served as the Principal Investigator for the Barcelona site for the CLOTBUST trial and as the Principal Investigator of the TUCSON trial. Dr. Andrei Alexandrov was the Principal Investigator of the CLOTBUST trial, study director of the TUCSON trial, serves on the speaker bureau of Genentech, Inc., and develops novel technologies under his patent on ultrasound-enhanced thrombolysis for stroke.

\section{References}

1 Derex L, Nighoghossian N: Intracerebral haemorrhage after thrombolysis for acute ischaemic stroke: an update. J Neurol Neurosurg Psychiatry 2008;79:1093-1099.

2 Khatri P, Wechsler LR, Broderick JP: Intracranial hemorrhage associated with revascularization therapies. Stroke 2007;38:431440.

3 Lou M, Safdar A, Mehdiratta M, Kumar S, Schlaug G, Caplan L, Searls D, Selim M: The HAT Score: a simple grading scale for predicting hemorrhage after thrombolysis. Neurology 2008;71:1417-1423.

4 Alexandrov AV, Molina CA, Grotta JC, Garami Z, Ford SR, Alvarez-Sabin J, Montaner J, Saqqur M, Demchuk AM, Moyé LA, Hill MD, Wojner AW; CLOTBUST Investigators: Ultrasound-enhanced systemic thrombolysis for acute ischemic stroke. N Engl J Med 2004;351:2170-2178.
5 Molina CA, Barreto AD, Tsivgoulis G, Sierzenski P, Malkoff MD, Rubiera M, Gonzales N, Mikulik R, Pate G, Ostrem J, Singleton W, Manvelian G, Unger EC, Grotta JC, Schellinger PD, Alexandrov AV: Transcranial ultrasound in clinical sonothrombolysis (TUCSON) trial. Ann Neurol 2009;66:2838.

6 Demchuk AM, Burgin WS, Christou I, Felberg RA, Barber PA, Hill MD, Alexandrov AV: Thrombolysis in brain ischemia (TIBI) transcranial Doppler flow grades predict clinical severity, early recovery, and mortality in patients treated with intravenous tissue plasminogen activator. Stroke 2001;32:8993.

7 Burgin WS, Malkoff M, Felberg RA, Demchuk AM, Christou I, Grotta JC, Alexandrov AV: Transcranial Doppler ultrasound criteria for recanalization after thrombolysis for middle cerebral artery stroke. Stroke 2000; 31:1128-1132.

8 Alexandrov AV, Burgin WS, Demchuk AM, El-Mitwalli A, Grotta JC: Speed of intracranial clot lysis with intravenous TPA therapy: sonographic classification and short term improvement. Circulation 2001;103:28972902.

9 Chernyshev OY, Garami Z, Calleja S, Song J, Campbell MS, Noser EA, Shaltoni H, Chen CI, Iguchi Y, Grotta JC, Alexandrov AV: The yield and accuracy of urgent combined carotid-transcranial ultrasound testing in acute cerebral ischemia. Stroke 2005;36:3237.

10 Tsivgoulis G, Sharma VK, Lao AY, Malkoff $\mathrm{MD}$, Alexandrov AV: Validation of transcranial Doppler with computed tomography angiography in acute cerebral ischemia. Stroke 2007;38:1245-1249. 
11 Saqqur M, Tsivgoulis G, Molina CA, Demchuk AM, Shuaib A, Alexandrov AV; CLOTBUST Investigators: Residual flow at the site of intracranial occlusion on transcranial Doppler predicts response to intravenous thrombolysis: a multi-center study. Cerebrovasc Dis 2009;27:5-12.

12 Saqqur M, Tsivgoulis G, Molina CA, Demchuk AM, Siddiqui M, Alvarez-Sabín J, Uchino K, Calleja S, Alexandrov AV; CLOTBUST Investigators: Symptomatic intracerebral hemorrhage and recanalization after IV rt-PA: a multicenter study. Neurology 2008; 71:1304-1312.

13 The NINDS rt-PA Stroke Study Group: Tissue plasminogen activator for acute ischemic stroke. N Engl J Med 1995;333:1581-1587.

14 Alexandrov AV, Grotta JC: Arterial reocclusion in stroke patients treated with intravenous tissue plasminogen activator. Neurology 2002;59:862-867.

15 Alexandrov AV, Wojner AW, Grotta JC: CLOTBUST: design of a randomized trial of ultrasound-enhanced thrombolysis for acute ischemic stroke. J Neuroimaging 2004; 14:108-112.

16 Barreto AD, Sharma VK, Lao AY, Schellinger PD, Amarenco P, Sierzenski P, Alexandrov AV, Molina CA: Safety and dose-escalation study design of Transcranial Ultrasound in Clinical SONolysis for acute ischemic stroke: the TUCSON Trial. Int I Stroke 2009;4:4248.
17 Wahlgren N, Ahmed N, Dávalos A, Ford GA Grond M, Hacke W, Hennerici MG, Kaste M, Kuelkens S, Larrue V, Lees KR, Roine RO, Soinne L, Toni D, Vanhooren G; SITS-MOST investigators: Thrombolysis with alteplase for acute ischaemic stroke in the Safe Implementation of Thrombolysis in Stroke-Monitoring Study (SITS-MOST): an observational study. Lancet 2007;369:275-282.

18 Lewis JR, Sauro J: When $100 \%$ really isn't $100 \%$ : improving the accuracy of small-sample estimates of completion rates. J Usability Stud 2006;1:136-150.

19 de la Ossa NP, Castellanos M, Jimenez M, Lopez-Cancio B, Dorado L, Guerrero C, Millan M, Serena J, Davalos A: Validation of the HAT-score scale as a predictor of hemorrhagic transformation in a large cohort of acute stroke patients treated with intravenous tPA. Stroke 2010;41:e30.

20 Tsivgoulis G, Saqqur M, Sharma VK, Lao AY, Hill MD, Alexandrov AV: Association of pretreatment blood pressure with tissue plasminogen activator-induced arterial recanalization in acute ischemic stroke. Stroke 2007;38:961-966.

21 Ribo M, Molina C, Montaner J, Rubiera M, Delgado-Mederos R, Arenillas JF, Quintana M, Alvarez-Sabín J: Acute hyperglycemia state is associated with lower tPA-induced recanalization rates in stroke patients. Stroke 2005;36:1705-1709.

22 Martini SR, Hill MD, Alexandrov AV, Molina CA, Kent TA: Outcome in hyperglycemic stroke with ultrasound-augmented thrombolytic therapy. Neurology 2006;67: 700-702.
23 Tsivgoulis G, Saqqur M, Sharma VK, Lao AY, Hoover SL, Alexandrov AV; CLOTBUST Investigators: Association of pretreatment ASPECTS scores with tPA-induced arterial recanalization in acute middle cerebral artery occlusion. J Neuroimaging 2008;18:5661.

24 Ibrahim MM, Sebastian J, Hussain M, AlHussain F, Uchino K, Molina C, Khan K, Demchuk AM, Alexandrov AV, Saqqur M; CLOTBUST Investigators: Does current oral antiplatelet agent or subtherapeutic anticoagulation use have an effect on tissue-plasminogen-activator-mediated recanalization rate in patients with acute ischemic stroke? Cerebrovasc Dis 2010;30:508-513.

25 Molina CA, Alexandrov AV, Demchuk AM, Saqqur M, Uchino K, Alvarez-Sabín J; CLOTBUST Investigators: Improving the predictive accuracy of recanalization on stroke outcome in patients treated with tissue plasminogen activator. Stroke 2004;35: 151-156.

26 Nordt TK, Klassen KJ, Schneider DJ, Sobel BE: Augmentation of synthesis of plasminogen activator inhibitor type-1 in arterial endothelial cells by glucose and its implications for local fibrinolysis. Arterioscler Thromb 1993;13:1822-1828.

27 Tsivgoulis G, Alexandrov AV, Sloan MA: Advances in transcranial Doppler ultrasonography. Curr Neurol Neurosci Rep 2009;1: 46-54. 